\title{
FLEXIBLE SYSTEMS FOR WEARABLE PHYSIOLOGICAL MONITORING APPLICATIONS
}

\author{
Sam Emaminejad ${ }^{1,2 *}$, Wei Gao ${ }^{1 *}$, Hnin Yin Yin Nyein ${ }^{l}$, Samyuktha Challa ${ }^{2}$, Ronald W. Davis ${ }^{2}$, and Ali Javey ${ }^{l}$ \\ ${ }^{1}$ Department of Electrical Engineering and Computer Sciences, University of California, Berkeley, CA, USA \\ ${ }^{2}$ Stanford Genome Technology Center, Stanford School of Medicine, Stanford, CA, USA \\ * Equal Contribution
}

\begin{abstract}
A flexible-system integration strategy is demonstrated which merges plastic based sensors that interface with the skin with silicon integrated circuits consolidated on a flexible printed circuit board for complex signal processing. As an example application, we present a flexible fully-integrated and wireless perspiration biosensor that accurately and simultaneously measures a panel of electrolytes and metabolites in sweat in real-time while calibrating the sensors' response against the change in skin temperature.
\end{abstract}

\section{INTRODUCTION}

Wearable biosensors have a significant role in the future of the healthcare as they facilitate real-time and non-invasive monitoring of an individual's health state. The implications of such wearable devices are far reaching and include various applications, such as fitness and athletic monitoring, as well as remote health monitoring of toddlers, elderly people and patients with disabilities or conditions that are in continuous need of a caregiver's attention. Currently commercialized wearable sensors are only capable of tracking an individual's physical activities and vital signs and do not provide insightful physiological information at molecular levels.

The advances in the field of flexible electronics have opened up the possibility of developing new classes of sensors that form intimate contact with human body and analyze previously underexplored physiologically rich sources of information (e.g. sweat, skin etc.). For these wearable biosensors to find application in clinical and translational medicine settings, an integrated-system approach must be adopted to ensure that the originated signals at the sensor level are accurately processed, calibrated against other influential parameters, and are seamlessly transferred to a display/storage unit or cloud servers online. Accordingly, on-site analog/digital signal processing and data transmission functionalities are required that interface the sensor unit. To this end, silicon-based integrated-circuit technologies should be utilized as they have already been optimized for low power and wireless consumer applications. The challenge remains in devising an integrated system which meets the mechanical constraints at the sensor level and the electrical constraints at the circuit level while providing a fully flexible solution that can be comfortably worn.

\section{FLEXIBLE INTEGRATED SYSTEMS}

Here, we devise a flexible-system integration strategy which merges plastic based sensors that interface with the skin with silicon integrated circuits (ICs) consolidated on a flexible printed circuit board (FPCB) for complex signal processing (Figure 1). This approach decouples the aforementioned stringent mechanical and electrical requirements, and at the same time exploits the strengths of the underlying technologies.

As an example application, we demonstrate a flexible fullyintegrated and wireless perspiration biosensor that accurately and simultaneously measures the main electrolytes (e.g. sodium and potassium) and metabolites (e.g. glucose and lactate) of sweat in real-time while calibrating the sensors' response against the change in skin temperature [1].
In this approach, the sensors are patterned and functionalized on a mechanically flexible polyethylene terephthalate (PET) substrate and the IC chips and other circuit components are assembled on a thin polyimide-based PCB (thickness $\sim 200 \mu \mathrm{m}$ ). The electrolyte sensors are potentiometric based and are realized with the aid of ion-selective membranes.

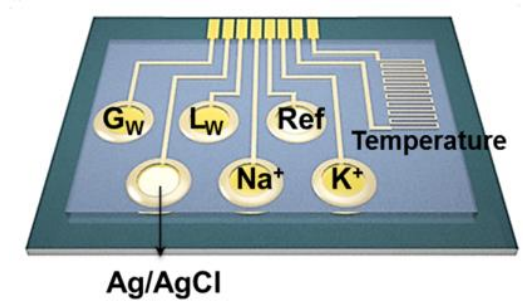

(a)

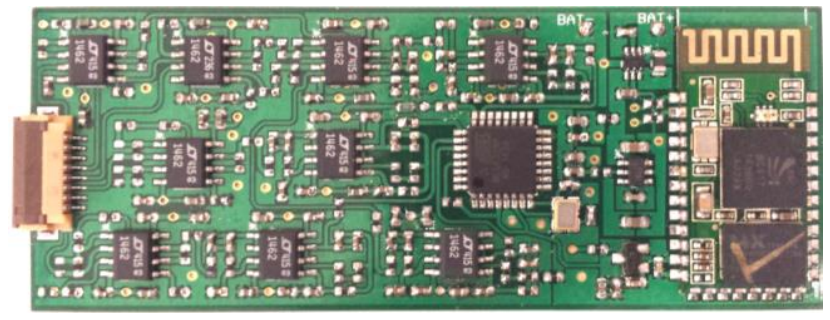

(b)

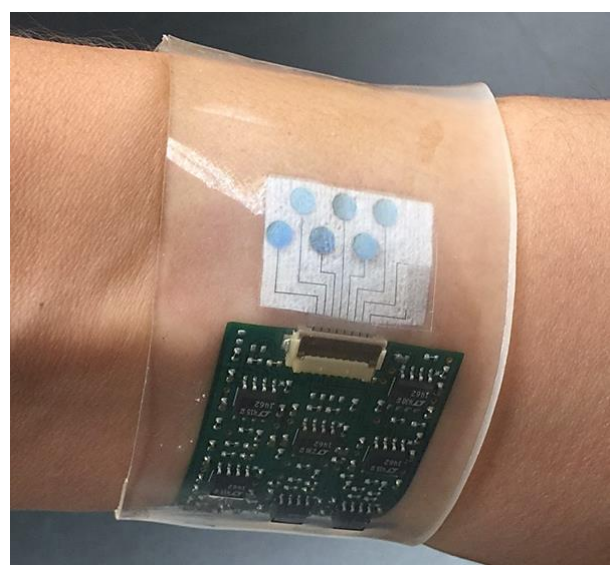

(c)

Figure 1: (a) Schematic of the sensor array (b) Top-view of a flattened flexible PCB. The PCB amplifies, filters, and calibrates the transduced signals. It then wirelessly transmits the data through Bluetooth to a custom-developed mobile application. (c) The fully flexible integrated platform where plastic-based sensors interface the flexible wireless $P C B$.

\section{RESULTS AND DISCUSSION}

As shown in Figure 2, the electrolyte sensors generate electrical voltage that is logarithmically proportional to the abundance of the respective electrolytes in sweat.

Solid-State Sensors, Actuators and Microsystems Workshop Hilton Head Island, South Carolina, June 5-9, 2016 


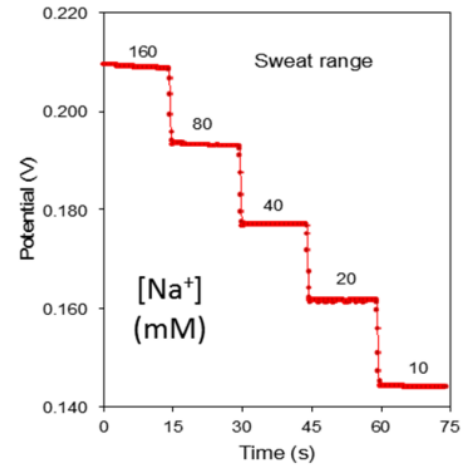

(a)

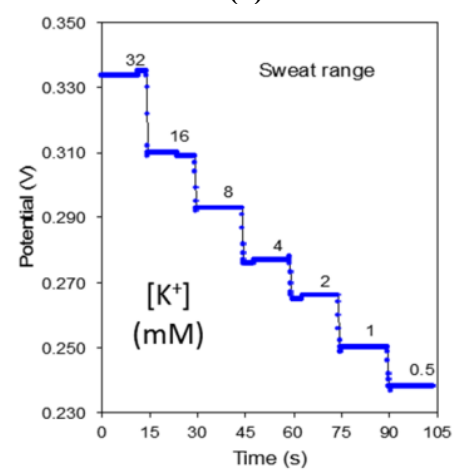

(b)

Figure 2: The potentiometric electrolyte sensor responses to the respective analyte solutions in phosphate-buffered saline (PBS) (a) sodium and (b) potassium sensors.

The metabolite sensors are enzymatic based and are functionalized with glucose oxidase and lactate oxidase (Figures 3a and $3 b b)$.

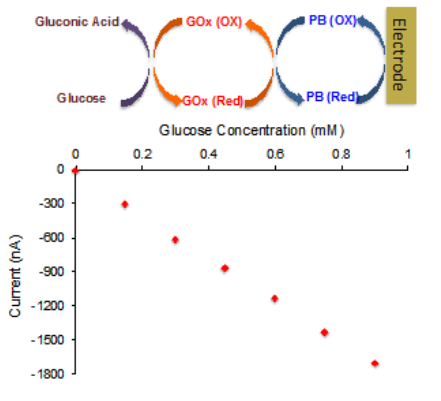

(a)

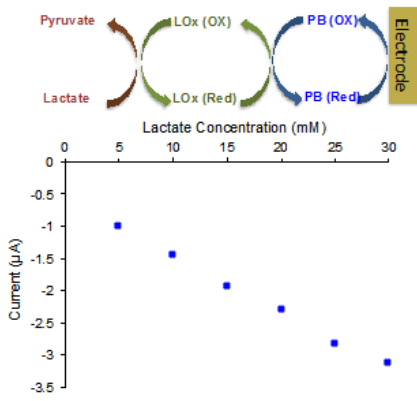

(b)

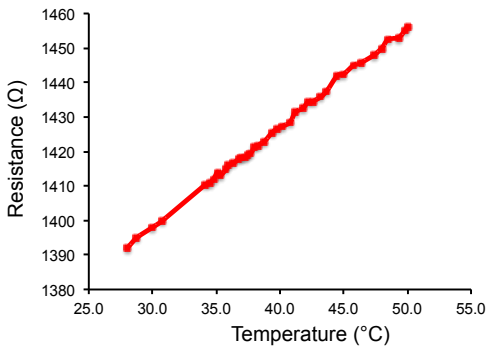

(c)

Figure 3: The enzymatic-based metabolite sensor responses to the respective analyte solutions in phosphate-buffered saline (PBS) (a) glucose and (b) lactate sensors. (c) The resistance response of the temperature sensor to temperature changes in PBS.
These enzymatic sensors generate electrical current that is linearly proportional to the abundance of the respective metabolites in sweat. Here, the temperature sensor information (Figure $3 \mathrm{c}$ ) not only serves as a useful marker of the thermal state of the individuals, but also is required to calibrate the enzymatic sensor readings against the skin temperature.

The form factor of this flexible platform allows for its placement on various body parts such as forehead and wrist. This platform is used to measure the detailed sweat profile of human subjects engaged in prolonged indoor and outdoor physical activities. Figure 4 illustrates the representative glucose and sodium readings from real-time perspiration monitoring on the forehead of a subject during stationary leg cycling.

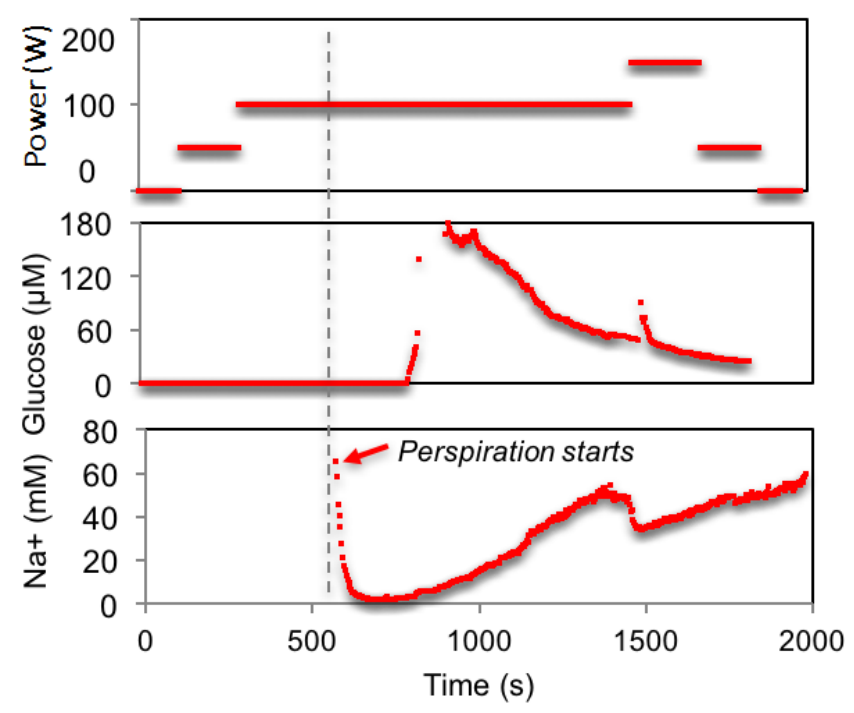

Figure 4: On-body real-time perspiration analysis during stationary cycling with varying power output. Representative sweat glucose and sodium readings are plotted (the accuracy of in-situ readings were verified through comparison with sweat samples collected at discrete time points)

We envision that this platform could be exploited or reconfigured for in-situ analyses of other biomarkers within sweat and other fluid samples to facilitate personalized and real-time physiological and clinical investigations.

\section{ACKNOWLEDGEMENTS}

The sensor design, characterization and testing aspects of this work were supported by the Berkeley Sensor and Actuator Center, and National Institutes of Health grant number P01 HG000205. The sensor fabrication was performed in the Electronic Materials (E-MAT) laboratory funded by the Director, Office of Science, Office of Basic Energy Sciences, Material Sciences and Engineering Division of the US Department of Energy under contract number DE-AC02-05CH11231.

\section{REFERENCES}

[1] W. Gao, S. Emaminejad, H. Y. Y. Nyein, S. Challa, K. Chen, A. Peck, H. M. Fahad, H. Ota, H. Shiraki, D. Kiriya, D.-H. Lien, G. A. Brooks, R. W. Davis, A. Javey. "Fully-integrated wearable sensor arrays for multiplexed in-situ perspiration analysis", Nature, 529, 509-514 (2016).

\section{CONTACT}

*A. Javey, ajavey@eecs.berkeley.edu 